This is the pre-peer reviewed version of the following article: Jox RJ (2020) Living will versus will to live? How to navigate through complex decisions for persons with dementia. Am J Bioethics 20;8:85-87. DOI 10.1080/15265161.2020.1781966. This article may be used for non-commercial purposes in accordance with Wiley Terms and Conditions for Use of Self-Archived Versions

\title{
Living will versus will to live? How to navigate through complex decisions for persons with dementia
}

\author{
Ralf J. Jox
}

Institute for Humanities in Medicine, Lausanne University Hospital and University of Lausanne, Lausanne, Switzerland

\section{Correspondence:}

Professor Ralf J. Jox

Institut des humanités en médecine, CHUV

Avenue de Provence 82

CH-1007 Lausanne, Suisse

Phone : +41 795566258

E-Mail : ralf.jox@chuv.ch

Word count : 1151 words

Ralf J. Jox is associate professor of medical ethics and of geriatric palliative care at the University of Lausanne, Switzerland. He is a trained neurologist, palliative care specialist, and philosopher. His research focus is decision making in situations of vulnerability. He has advised governments on advance care planning and end-of-life decision making. 


\section{Living will versus will to live? How to navigate through complex decisions for persons with dementia}

In her target article, Emily Walsh argues that persons with moderate-to-severe dementia undergo a cognitive transformative experience (Paul 2016), which is why their new preferences would morally outweigh their pre-dementia preferences (Walsh 2020). She thus purports to philosophically confirm a clinical reluctance towards respecting advance directives in a state of dementia. Yet, her argument collapses because it is built on flawed understandings of dementia, personal autonomy, and clinical practice. I will briefly explain each of these three misconceptions and suggest alternative ways to think about this problem and more adequate approaches for clinical practice.

Fully respecting persons with dementia begins with being realistic about the disease and its underlying pathology (Arvanitakis, Shah, and Bennett 2019). Dementia is a syndrome commonly caused by wide-spread, progressive neurodegeneration in the cerebral cortex, gradually disrupting multiple cognitive, affective and volitional functions, including memory, a stable and reflective self-image, as well as longreaching biographical intentions. Consequently, persons with dementia in advanced stages lose the condition of possibility of any cognitive transformative experience. The experiences that they make are largely disconnected from each other, as well as from the experience they had before the onset of dementia. If something is disconnected, however, it cannot logically be transformed. For experiences to have a transformative effect, they have to be stored, remembered and compared to each other. This is why all the examples that the author advances vanish if memory is disrupted: if you have a new religious experience, but are unable to keep it in mind, it will not transform your life. Since dementia is not a transformative experience, it does not cause a change of preferences. What happens in dementia is that some preferences are lost, while others may emerge (or remain). Both, however, are preferences on different levels: The preferences we lose with dementia are the more abstract, long-reaching ones that are rooted in our values, biography and self-image (e.g. the moral preference to be a vegetarian), while the preferences that emerge are concrete, short-term preferences rooted in our immediate perception (e.g. the wish to eat meat that looks and smells deliciously). 
This leads me to the second misconception in the article of Walsh, which is the misconception about personal autonomy. The text seems to insinuate that in dementia autonomy is either lost or preserved (and just the preferences change). Yet, a more differentiated and richer account of autonomy considers at least two constituents: being able to realize one's wishes and preferences with regard to external objectives and being able to aspire to self-determined internal values and attitudes (the latter is closer to the Kantian notion of autonomy). This reflects Harry G. Frankfurt's insight that human free will not only includes first-order desires (the preferences we have) but also secondorder desires (the preferences we want to have) (Frankfurt 1971). Persons with moderate-to-severe have lost the latter but partially preserved the former. Furthermore, autonomy as a capacity is always task-dependent: while persons with dementia may not have the necessary cognitive capacities to decide autonomously about a hospitalization, surgery, or antibiotic treatment, they may well be able to autonomously decide about questions of daily care, meals, activities or clothing. For this reason, however, expressions of a presumed will to live cannot be understood as revocation of their living wills (advance directives), when they do not have any more the prerequisite level of autonomy with regard to major health care decisions that they used to have when they had written the directive in the first place (Jox 2014). So, what should we do in clinical practice: are we left with the dilemma of either disregarding the advance directive or disregarding current expressions that may seem to contradict these directives?

The answer to this question points to the third misconception in Walsh' article, that about clinical practice. Clinicians are familiar with balancing the principles of biomedical ethics, in particular the principles of beneficence and non-maleficence versus the principle of respect for patient autonomy. When a patient with moderate-tosevere dementia develops pneumonia and the advance directive seems to contradict the behavioral signs of a supposed will to live, they have to specify and balance precedent autonomy and beneficence. In doing so, the following considerations may be helpful. For precedent autonomy, the current gold standard is to embed advance directives into a comprehensive, systemic model of advance care planning (ACP) that includes a longitudinal process of structured facilitation by qualified professionals and a regional implementation to ensure that the documents are adequately retrieved, understood and respected (Rietjens et al. 2017). Their effectiveness to enhance goal-concordant care has 
been established, including in the population with dementia (Houben et al. 2014, Kelly et al. 2019).

When ACP is specifically tailored to the situation of dementia (Bosisio et al. 2018), it has to include a thorough information about the course and implications of dementia. The anticipatory decisions that are made in ACP are not only based on the imagination how life with dementia would feel like, but also on time-transcending personal values and concerns for others. Moreover, ACP allows to anticipate and discuss the potential conflict between the advance directive and future behavioral expressions. The person can thus express the modal preference whether she wants to prioritize the former or the latter, which actually enhances the chance that the advance directive can be applied and followed (Schoene-Seifert et al. 2016).

We therefore need to develop dementia-specific ACP programs that involve professionals experienced in dementia care as well as decision aids and advance directive documents that consider the specificities of this illness. Such dementia-specific ACP should be offered soon after the diagnosis has been established and communicated. The fact that dementia is diagnosed increasingly early using imaging or laboratory biomarkers does in fact widen the window of opportunity when persons with dementia still retain decision-making capacity for major health care decisions.

Such evidence of precedent autonomy has to be balanced against considerations of the current and future wellbeing of the person with moderate-to-severe dementia. If the person seems to enjoy the activities of daily life, it is undoubtedly a sign of current wellbeing. Its moral weight increases if the observed behavior is consistent over time, concordant with previous behavior, and interpreted consensually by all caregivers (what I call the $3 \mathrm{C}$ 's). Nevertheless, signs of current wellbeing neither guarantee that the same kind of wellbeing will be preserved after a major hospital treatment nor do they allow to conclude a will to live. Concluding intentions from emotions would be a psychological fallacy. In fact, we know from many cognitively intact elderly people that they refuse life-sustaining treatment even though (or maybe because) they feel content and satisfied with their life. If the caregivers of persons with dementia meticulously apply these considerations in order to balance precedent autonomy in the form of ACP with a careful interpretation of current behavioral expressions, they will be able to responsibly navigate through these complex decisions without getting trapped in a dilemma. 


\section{References:}

Arvanitakis, Z., R. C. Shah, and D. A. Bennett. 2019. "Diagnosis and Management of Dementia: Review." JAMA 322 (16):1589-1599. doi: 10.1001/jama.2019.4782.

Bosisio, F., R. J. Jox, L. Jones, and E. Rubli Truchard. 2018. "Planning ahead with dementia: what role can advance care planning play? A review on opportunities and challenges." Swiss Med Wkly 148:w14706. doi: 10.4414/smw.2018.14706.

Frankfurt, HG. 1971. "Freedom of the will and the concept of a person." The Journal of Philosophy 68 (1):5-20.

Houben, C. H., M. A. Spruit, M. T. Groenen, E. F. Wouters, and D. J. Janssen. 2014. "Efficacy of Advance Care Planning: A Systematic Review and Meta-Analysis." J Am Med Dir Assoc 15 (7):477-489. doi: 10.1016/j.jamda.2014.01.008.

Jox, RJ. 2014. "Revocation of Advance Directives." In Advance Directives. International Library of Ethics, Law, and the New Medicine 54, edited by P. Lack, N. Biller-Andorno and S. Brauer, 73-90. Heidelberg New York: Springer.

Kelly, A. J., T. Luckett, J. M. Clayton, L. Gabb, S. Kochovska, and M. Agar. 2019. "Advance care planning in different settings for people with dementia: A systematic review and narrative synthesis." Palliat Support Care 17 (6):707719. doi: 10.1017/S1478951519000257.

Paul, L.A. 2016. Transformative Experience. Oxford: Oxford University Press.

Rietjens, J. A. C., R. L. Sudore, M. Connolly, J. J. van Delden, M. A. Drickamer, M. Droger, A. van der Heide, D. K. Heyland, D. Houttekier, D. J. A. Janssen, L. Orsi, S. Payne, J. Seymour, R. J. Jox, I. J. Korfage, and Care European Association for Palliative. 2017. "Definition and recommendations for advance care planning: an international consensus supported by the European Association for Palliative Care." Lancet Oncol 18 (9):e543-e551. doi: 10.1016/S1470-2045(17)30582-X.

Schoene-Seifert, B., A. L. Uerpmann, J. Gerss, and D. Herr. 2016. "Advance (Meta-) Directives for Patients with Dementia who Appear Content: Learning from a Nationwide Survey." J Am Med Dir Assoc 17 (4):294-9. doi: 10.1016/j.jamda.2016.01.014.

Walsh, E. 2020. "Cognitive Transformation, Dementia, and the Moral Weight of Advance Directives." Am J Bioethics forthcoming. 